\title{
Application of Industrial Moulding Design in the New Products of Series track-mounted Directional Drilling Rigs
}

\author{
Ke Yao ${ }^{a}$, Zhijun Shi ${ }^{b}$, Peng Fang ${ }^{c, k}$, Baoshan Sun ${ }^{d}$, Dong $\mathbf{L i}^{\mathrm{e}}$ \\ Xi' an Research Institute Co., Ltd of China Coal Technology \& Engineering Group Corp, Xi' an, China \\ ayaoke@cctegxian.com, ${ }^{\mathrm{b}}$ shizhijun@cctegxian.com, ${ }^{\mathrm{c}}$ fangpeng@cctegxian.com, \\ d sunbaoshan@cctegxian.com, ${ }^{\mathrm{e}}$ lidong@cctegxian.com \\ *corresponding author
}

Keywords: industrial moulding design; directional drilling rig; track-mounted; application

\begin{abstract}
As a kind of design technique, industrial moulding design has been applying in all kinds of production design, especial the new products. Taking the ZDY Directional Drilling Rigs as a example, the paper gives a detailed description of application and research of industrial moulding design in developing and design of ZDY directional drilling rigs. The results of production and application indicate that industrial design on modeling plays a very important role in improving operate comfort and appearances of drilling rigs.
\end{abstract}

\section{Introduction}

The technology gap between chinese coal mine drilling rig manufacturer and international counterparts is narrowing, but for the industrial design, prevalence concept such as do not need, no one care, inconvenient to use, as well as inadequate investment in industrial design and some other problems, they all made the product image and branding all still behind international leader company. So, through industrial design to enhance the product image of coal drilling rig is the inevitable choice to reduce this gap.

The paper based on the above background combined with the national "twelfth five-year" science and technology major project "technology and equipment of middle and hard coal seam high power directional drilling rig" did a new exterior design and prototype trial for two new directional drilling products of the ZDY series and explored the serialized designing of directional drilling rig.

\section{Styling and design}

Drilling rig design belong to the category of industrial design, which can not only limited to the exterior shape, color and decoration, we must consider the function, structure, material, ergonomics and other factors, must fellow the principle of "function first, form second", modeling design is not designer free to improvise. Excellent rig product not only to gradually improve function and reliability to meet the market demand, but also should consider the user's focus on the emotional needs and aesthetic requirements and operating experience, enabling users to simply and comfortable operation.

The directional drilling rig machines produced from Xi'an Research Institute of CCTEG are mostly used overall crawler-type which is above size smaller than below size reflects the stable atmosphere of product. Generally, this shape can lower the visual center of heavy machinery, especially for the deep hole directional drilling rig this design style can make crawler drilling rig has a sense of stability because of it looks like firmly noted in the ground. Coating whole machines always use more bright and high-contrast color matching system to improve the visual senses and strengthen the heaviness of the product. While, to the size and height of the machine itself, this form of design is more appropriate for practical requirements of engineering and safety of user, so the integral form design of drilling rig must on the basis of original style and features of drilling rig 
machines produced by Xi' an Research Institute of CCTEG.

First of all, we analysed domestic and foreign related products, collected the picture of benchmarking product produced from Industrial Mining Equipment and Atlas, then we analysed the work environment, appearance and coating technology of these machines. Finally combined with the present situation and future development trend of drilling rig, using modeling design theory and ergonomic to argument and improve the structure, layout, operation and argumentation of drilling rig, then analysis existing problem of the existing product such as is that the instrument in console area easy to observe; is that the zoning of console area compliance with user's operation and cognitive features; whether the operational information is easy to understand; is that the distance, high and size of handles accord with aesthetic principle and easy to control? These demonstration and analyzes laid the foundation for modelling design and also basically determines the design requirements and objectives.

Through design activities to ensure the morphology, coating and surface materials of drilling rig we decided the product style of Xi' an Research Institute of CCTEG, that is heavy and stable, simple and practical, beautiful and delicate. At the same time, preliminary create product style and brand image, through the modeling of drilling rig design reflects the "people-oriented" design concept, implement the security, convenience, reliability and efficiency of operation requires to form the product design system and formation of Xi' an Research Institute of CCTEG. Also we hope that the modelling design can make brand products have a great improve in appearance and strengthen the development of brand strategy in the end.

The whole process of design include determining the basic shape, painting style and complete shape and several important steps of detail design, specific steps in figure 1 . Throughout the design process, the program was assessed several times, after constantly improve and perfect finally achieved the perfect requirement for product design.

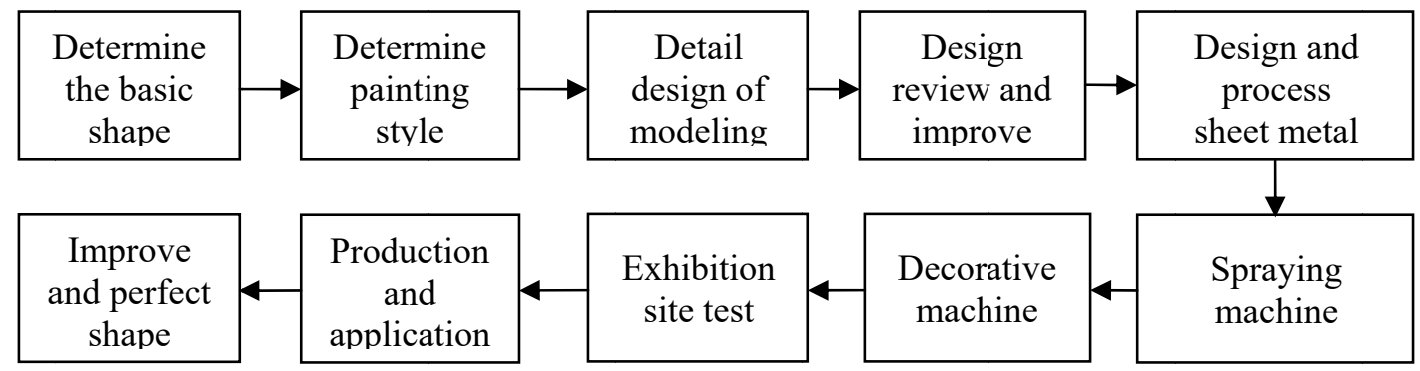

Figure 1 Moulding design and application course

\section{Painting style and color matching}

After years of research and application the drilling rig of Xi' an research institute of CCTEG has already formed a certain traditional features and product types, different kinds of drilling rig has external different structure. According to the structural type and product positioning, different original products has different color style and basically has the features of product image series, such as split drilling rig with red as the main color, some black with red, grand engineering drilling rig with yellow as the main color and crawler drilling rig with white as the main color.
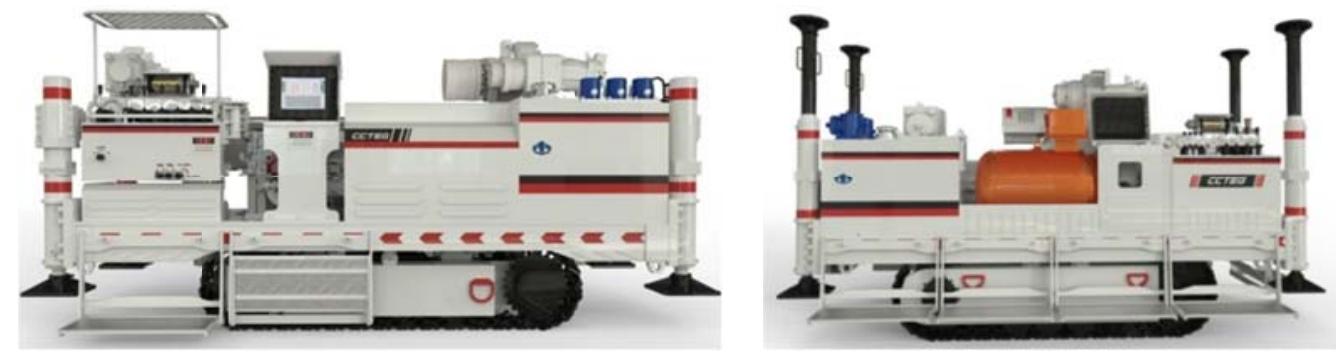

Figure 2 The moulding of directional drilling rigs

Through consideration we believe the color of drilling rig should take into account the function 
and safety factors and even environmental factors, also it must be reflect the enterprise culture and concept of product image. We continue use the former style of crawler drilling rig made from Xi'an research institute of CCTEG, and on this basis established a complete color scheme, that is overall in white with red and black decoration strip, local stiffener and hollow out are based on horizontal stripes, straight lines to the main and curve to supplement. On the whole, drilling rig reflect stable, simple, elegant and make enterprise logo better into drilling rig color matching system.

In the color system, accounting for 80percent of white and black accounted for 10percent or more, red is mainly used for the edge, this color collocation can play the role of striking warning in complex construction environment. Because of the sense of black can make a person feel sedate and the color of black with a strong soil resistance, so the chassis and connecting parts of drilling rigs are sprayed into the black, at the same time, as the connecting parts black can also effectively split the other two visually coating. Designed drilling rig modelling appearance is shown in figure 2 .

\section{The main modelling and detail design}

The drilling rig with overall crawler structure including power head, feeding device, gripper, cooler and so on. Due to the excessive structural components, the appearance of drilling rig should be uniform, chiselled, it must overcome the stiff and clumsy feeling of inherent mechanical products. In addition, for key components it must combine with overall effect to design, make its form a complete visual form. In the process of design, several original structure was necessary adjustments many times. The parts including pressure gauge, thermometer, filters and flowmeter and other hydraulic accessories, they all be replaced by the product which better appearance design and surface quality treatment.

\subsection{Master operational console}

Master operational console for the directional drilling rig is the key part in human-computer interaction links, design of this part need to be fully combined with human-computer interation principle. Designed master operational console modelling appearance is shown in figure 3.

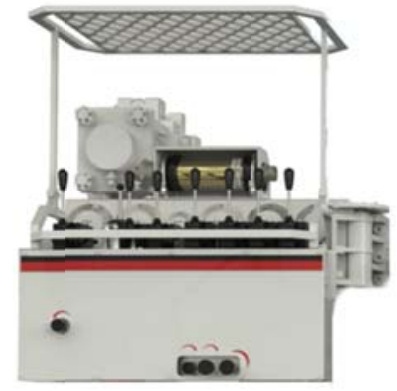

Figure 3 The moulding of master console

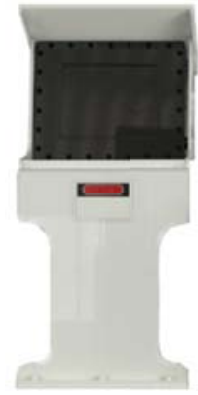

Figure 4 The moulding of computer

Black and red decorative stripe on the front panel enhanced the visual effect, operational instruction of round handwheel use the way that text with pictures, through changing the thinkness of the arrow to reflect the change of parameters can echoes with text. Curved armrest and handles on the top of console facilitate the rotation of the console and can prevent the lateral accidentally touch to handle, improve the safety and convenience. The fence at the top of the master operational console use polyline elements and hollow out pattern to match the neat style of whole machine and the hollow in the both ends make the overall style feel more beautiful, lightness and unified, also the grid model of the fence can not only protect console but also does not affect the upward gaze. To avoid the tubing below console looks messy we designed a plate with horizontal decoration stripe below the console, the stripe can make the plate feel more marrow in visually.

\subsection{Explosion-proof computer}

Explosion-proof computer is an important component, and lay in the position conducive to observing and operating with necessary protection package, the computer is installed in the way of a 
fixed inclination angle, which could effectively improve the comfort for the operator observing the computer screen; the lower part of the supporting base uses a new-shaped structure, which is similar to the model of podium and in the stable aesthetics of symmetrical design, and exposes the steering recognition observation hole of the electrical machinery, there is trough in the left side of the computer for easily installation and operation. The modeling of the computer is shown in Figure 4.

\subsection{Auxiliary operating platforms and cooler}

Auxiliary operating platforms and cooler are arranged between the rig body and the vehicle platform so that these could be operated and maintained easily; two manual multi-valve cylinder is mainly used to operate the rig to stably lifting up and down the hydro-cylinder and adjusting the pitch angle of the feeding device. The operating platforms must be easy to identify during operation and also to prevent accidents such as accidental collision and unexpected drop. The design of this part wrap and decorate both the auxiliary operating platforms and cooler with the design program of the whole rig. The design uses three-fold style model to enhance the third dimension and sense of hierarchic of the box structure by changing the physical dimensions. In order to improve the degree of recognition of the vice operating handle, the designed indicating text of nameplate in this position should be corresponded with the joystick, so that it could avoid malfunctions effectively. The outlet hole in the position of the cooler can improve the aese of the cooling water pipe installation and disassembly, and the under stenciled design would facilitate the heat dissipation and enhance practical function. The overall modeling is shown in Figure 5.

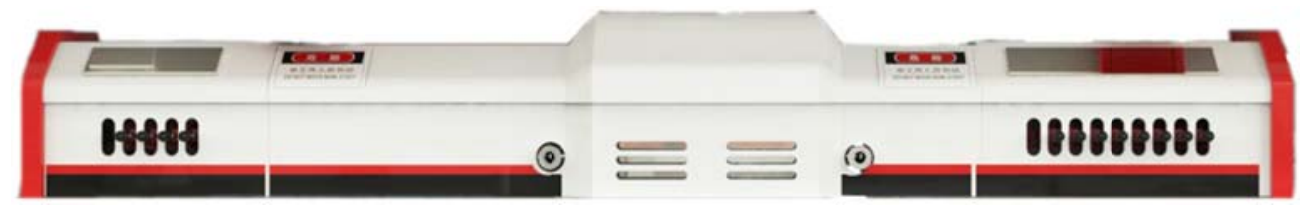

Figure 5 The moulding of auxiliary operating floor and cooling apparatus

\subsection{Decoration of electrical machinery guard plate}

Electrical machinery is the original power of equipment. We must consider the ventilation and heat dissipation during the normal operation, and prevent much water splashing and soaking from top. The design style between the electrical machinery guard plate and the bottom guard board must be echoed and play the role of shielding for the electrical machinery, so that the overall effect would be more beautiful.

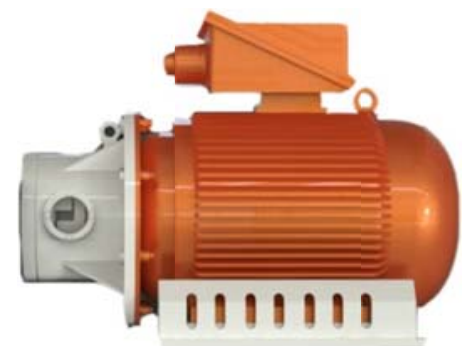

Figure 6 The moulding of motor guard board

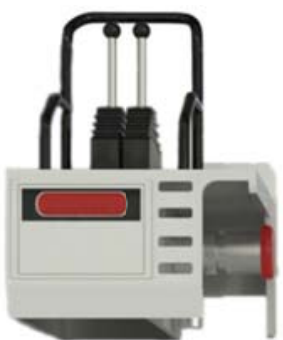

Figure 7 The moulding of walking console

Fringe oblique holes have the effect of cooling and decoration, and horizontal stripes appear stable and maintain the unity style of the whole machine. Otherwise, the model arranged like that would make the cooling air generated by the electrical machinery cool the pump in the passage of the sheet metal and hydraulic conduit at the bottom of the console, and the air would not blow to the drilling personnel, which could improve the comfort of operation. The overall decoration effect of the electrical machinery guard plate is shown in Figure 6.

\subsection{Walking console}

The design of walking console aim to make operate control handle be reliable and safe to make sure that user can control the speed and direction of drill travel. In order to improve the continuity 
of the product, walking console finally designed be a fixed structure set up on the rear end of tank by screws, so the modeling design requiring compact and easy to operate.

Beam can be used to withstand the weight of operator's hand, when drill is working operator can put hands on it to make operation more effort and easy, the design is full of humanness, even the tunnel uneven can also achieve good control of drilling rig walking. Emergency stop button arranged in the right side of walking console with a close distance to user's hand, in the form of half surrounded to prevent accidental touch and can also taking into account the need for rapid response in emergency situation. Walking console overall modeling results are shown in figure7.

\section{Design processing and post processing}

\subsection{The design and processing of sheet metal}

The connection, installation, rigidity, strength, processing technology, spray and painting or other issues must be considered in the process of sheet metal design, sometimes in order to install support structure conveniently designer must to negotiate with the product development personnel to adjustment the installation location of some component, for practical functional requirements, such as easy disassembly but hard to damage and deformation, the number of sheet metal should be as less as possible and minimize the use of screws, the latch structure is the most convenient way to apply on site, because the losing of screw will not affect the use of sheet metal. The sheet metal design of bending, shutter and sediment in tank, they all need to fully considered the maneuverability of the actual processing, some size of shutter and sediment in tank must customize punching mold to realize.

\subsection{The spray of whole machine}

Before painting, all exposed machined part surfaces should be inspection and evaluation to decide the part which need to be burnish including weld, edge and sharp corner and the extent of them. Then, began to crafted polished, after polish, painting putty to make each piece smooth, then, in accordance with design drawing to paint in paint room.

\subsection{Decorative machine assembly}

In order or better strengthen the sense of stability and brand in the paint, we weld the decorative sheet to the edge of the base, so that the base appears stable and increases slightly smart. The grip hook with red color play the role of embellishment. Handling precautions, all kinds of warning signs and red decorative reflective warning bar could improve the safety of the device but also add luster for the rig. We paint the chuck of the moving parts and the rig body with the bright red, and performance the product itself moving and the way of practices well by the ribbon style full of decorative art language.

\section{Production applications and improved styling}

After the completion of two research prototype trial, the rig has participated in the Summit of the coal industry and Equipment Exhibition in Beijing in 2012, Coal Industry Equipment Exhibition in Xinjiang in 2013 and other several large equipment exhibitions. It shows that products would be highly recognized by the market users after industrial designing. Meanwhile the prototype has been experienced the site industrial test and long practical application in the coal mines affiliated the Shanxi Coal, and showed the whole nice appearance, operation and maintenance of safe, simple and comfortable, beautiful decorations and eye-catching colors, the overall sense of heavy stable, practical and simple, beautiful and delicate.

\section{Conclusion}

(1) Industrial design is design activity combination of technology and art, and the constantly harmonized integrated design process between the utilitarian function and the beauty sense of 
decoration. The processes involve structural design, modeling design, sheet metal processing, painting, assembly and decoration processing and other necessary processes. There are important links with coordination between the various processes.

(2) A good industrial product need a team to complete, the team must include the members who work many years in research and development of equipment and the personnel of product modeling engineers, machining and manufacturing engineers and even mould engineers. Good product is the result of joint efforts from the research team with different professional knowledge, working experience innovative vision and philosophy.

(3) The excellent product should innovate from design method and design process. To higher level development of industrial design, R\&D personnel need to has the same understanding with customers in product design, market consumption and competitive, only in this way can produce the product that consumer truly need and satisfy.

\section{Acknowledgements}

Important national science and technology projects (2011ZX05041-001).

\section{References}

[1] Haiyun Wang. New Exploration of Industrial Modeling Design Process[J]. Equipment Manufacturing Technology, 2015(5) : 208-210.

[2] Jinyan Zhu. Natural Shape and Industrial Modeling[J]. Art and Design, 2011(5) : 210-212.

[3] Chunlin $\mathrm{Yu}$, Renping Hang, Fufeng Chen. Power automation device industrial molding design[J]. Huadian Technology, 2017(5) : 36-38,41.

[4] Minji Zhu, Xingfang Wang. Discussion on industrial modeling of mine vehicles[J]. Mining Technology, 2014(5) : 37-38,54.

[5] Wei Chen. Discussion of Design in GPS Production Shape[J]. Development \& Innovation of Machinery \& Electrical Products, 2011(5) : 46,58-59. 